\title{
Centrifugal microfluidics for sorting immune cells from whole blood
}

Zeta Tak For Yu ${ }^{\text {a\# }}$, Jophin George Joseph ${ }^{\text {a, b\# }}$, Shirley Xiaosu Liu ${ }^{\mathrm{a}}$, Mei Ki Cheung ${ }^{\mathrm{a}}$, Parker James Haffey ${ }^{\mathrm{a}}$, and Jianping $\mathrm{Fu}^{\mathrm{a}, \mathrm{c}, \mathrm{d}, \mathrm{e} \text { * }}$

${ }^{a}$ Department of Mechanical Engineering, University of Michigan, Ann Arbor, Michigan 48109, USA; ' ${ }^{\mathrm{b}}$ Department of Chemical Engineering, Indian Institute of Technology Hyderabad, Kandi, Sangareddy, Telangana, India 502285; ${ }^{\mathrm{C}}$ Department of Biomedical Engineering, University of Michigan, Ann Arbor, Michigan 48109, USA; ${ }^{\mathrm{d}}$ Department of Cell and Developmental Biology, University of Michigan Medical School, Ann Arbor, MI 48109, USA; ${ }^{\mathrm{e}}$ Michigan Center for Integrative Research in Critical Care, University of Michigan, Ann Arbor, MI 48109, USA.

\#These authors contributed equally to this work.

*Corresponding author (Email: jpfu@umich.edu)

(C) 2017. This manuscript version is made available under the Elsevier user license http://www.elsevier.com/open-access/userlicense/1.0/ 


\begin{abstract}
Sorting and enumeration of immune cells from blood are critical operations involved in many clinical applications. Conventional methods for sorting and counting immune cells from blood, such as flow cytometry and hemocytometers, are tedious, inaccurate, and difficult for implementation for point-of-care (POC) testing. Herein we developed a microscale centrifugal technology termed Centrifugal Microfluidic Chip (CMC) capable of sorting immune cells from blood and in situ cellular analysis in a laboratory setting. Operation of the CMC entailed a blood specimen layered on a density gradient medium and centrifuged in microfluidic channels where immune cell subpopulations could rapidly be sorted into distinct layers according to their density differentials. We systematically studied effects of different blocking molecules for surface passivation of the CMC. We further demonstrated the applicability of CMCs for rapid separation of minimally processed human whole blood without affecting immune cell viability. Multi-color imaging and analysis of immune cell distributions and enrichment such as recovery and purity rates of peripheral blood mononuclear cells (PBMCs) were demonstrated using CMCs. Given its design and operation simplicity, portability, blood cell sorting efficiency, and in situ cellular analysis capability, the CMC holds promise for blood-based diagnosis and disease monitoring in POC applications.
\end{abstract}

\title{
KEYWORDS
}

Centrifugation; Microfluidics; Blood cell sorting; Immune Cells. 


\section{INTRODUCTION}

Status of human immune system can reflect health, aid disease diagnosis, and facilitate drug treatment efficacy monitoring [1-3]. Each type of immune cells (or white blood cells, WBCs) mounts a specific defense mechanism against diverse invasions. The five main immune cells in circulating blood: neutrophils (CD15+), eosinophils (CD193+), basophils (CD123+), lymphocytes (CD3+ for T cells, CD19+ for B cells, and CD56+ for natural killer cells or NK cells), and monocytes (CD14+) are related to bacterial infection, parasite infection, allergic reaction, adaptive immunity, and phagocytosis, respectively. Neutrophils, eosinophils, and basophils are collectively called polymorphonuclear (PMN) cells, whereas lymphocytes and monocytes are collectively termed peripheral blood mononuclear cells (PBMCs). Importantly, the number of different immune cells in blood circulation has both prognostic and diagnostic values for diseases such as cancer and HIV [4].

In the clinic, complete blood count $(\mathrm{CBC})$ is a common blood test that reports the count of immune cell subpopulations. Automatic CBC can be achieved by flow cytometric instruments, which, however, are expensive due to sophisticated electrical and optical methods used for characterizing cell types. Moreover, flow cytometry cannot distinguish abnormal cells by morphology, nor can it confirm the existence of pathogens. Manual CBC can be conducted on a blood smear. Although manual counting allows identification of abnormal cells and pathogens, it requires an experienced technician to distinguish subtle differences among chromatically stained WBC subpopulations, thus making it error prone. Both conventional automatic and manual $\mathrm{CBC}$ methods process a mixture of target immune cells over abundant red blood cell (RBC) background, making such methods laborious and time-consuming [1]. 
Blood centrifugation over a density gradient medium (DGM) such as the Ficoll or Percoll solution is an efficient technique to separate and sort blood cells into different layers according to their inherent density differences (Fig. 1a) [1]. In blood centrifugation over a DGM, different blood cells in a bulk fluid migrate to different equilibrium positions according to density differentials in a rotational motion (Fig. 1b). Through miniaturization, microfluidic centrifugation systems that incorporate a DGM have been successfully developed, which have been shown to be capable of separating whole blood into multiple cell layers. However, existing microfluidic centrifugation systems involving a DGM still fall short of integrating both blood cell sorting and in situ cellular imaging and enumeration [5-8]. Specifically, designs of existing microfluidic centrifugation systems, with complex microfluidic networks to handle blood and the DGM, are suboptimal for integration with optical detection due to large device thickness, bulky setups, intricate units, and complicated operations that prohibit optical accessibility $[9,10]$. Thus, for existing microfluidic centrifugation systems using the DGM, cellular analysis is commonly achieved by an external platform to collect blood cells after centrifugation for downstream cellular analysis.

Herein, we report the development of a single-layer, simple-configuration Centrifugal Microfluidic Chip (CMC) that is compatible with in situ multi-color fluorescence microscopy $[11,12]$. The CMC enables efficient and convenient sample manipulation, blood separation operation, and quantitative cellular analysis of minimally processed whole blood. We investigated and optimized various aspects of the CMC, including surface passivation, structural design, operational control, cell viability, separation stability, and multi-color fluorescence imaging, in order to achieve efficient blood cell sorting and on-chip cellular analysis and enumeration. Given its design and operation simplicity, portability, blood cell sorting efficiency, 
and in situ cellular analysis capability, the CMC holds promise for blood-based diagnosis and disease monitoring in point-of-care (POC) applications.

\section{MATERIALS AND METHODS}

2.1. Chip fabrication. CMCs were fabricated using soft lithography [11, 13-15]. CAD designs were printed out as transparency masks (CAD/Art Services, Inc., Bandon, OR). Channel patterns on Si wafers were fabricated by patterning a layer of negative photoresist, SU8 (Microchem, Westborough, MA), with a thickness of about $100 \mu \mathrm{m}$, using photolithography. The Si wafer was further coated conformably with a 0.5 - $1 \mu \mathrm{m}$ thick Parylene $\mathrm{C}$ dielectric layer at $690{ }^{\circ} \mathrm{C}$ and $30 \mathrm{mTorr}$ (Specialty Coating Systems, Indianapolis, IN).

Based on the same planar design, four types of CMCs, with varying polydimethylsiloxane (PDMS; Dow Corning, Ellsworth, Germantown, WI) channel-layer thickness and number of glass slides bounding PDMS layers, were fabricated using the Si mold by soft lithography (see Fig. 5a for illustrations of different CMC configurations) [11, 13-15]. For noglass, bottom-glass, and glass-sandwich CMCs with a $2 \mathrm{~mm}$ thick PDMS channel-layer, PDMS prepolymer with a 10:1 monomer to curing agent ratio was poured over the Si mold and degassed. The PDMS channel-layer thickness was controlled by squeezing PDMS prepolymer against microscope slides. For fabrication of glass-sandwich CMCs with a $400 \mu \mathrm{m}$ thin channellayer membrane, PDMS prepolymer was spin-coated on the Si mold at a spin speed of $500 \mathrm{rpm}$. After baking at $80^{\circ} \mathrm{C}$ for $30 \mathrm{~min}$, PDMS layers were peeled off from the Si mold, cut into cubes, and punched with $0.5 \mathrm{~mm}$ diameter holes before cleaning and treating with air plasma (Femto Science, Gyeonggi-Do, Korea). For no-glass CMCs, 2 mm thick PDMS channel-layers were bonded to another $2 \mathrm{~mm}$ thick blank PDMS. For bottom-glass and glass-sandwich CMCs, 
PDMS channel-layers were first bonded to glass slides (Thermo Fisher Scientific, Waltham, MA) precoated with a $50 \mu \mathrm{m}$ thick PDMS layer. A thin glass coverslip (Thermo Fisher Scientific) was then bonded on top of the PDMS channel-layer to transform a bottom-glass to a glasssandwich CMC. CMCs were further baked at $80{ }^{\circ} \mathrm{C}$ overnight to complete PDMS polymerization and strengthen irreversible bonding between PDMS and glass surfaces.

2.2. Blood collection and treatment. Blood collection was approved by the Institutional Review Board (IRB) of the University of Michigan, Ann Arbor. Informed consent was obtained from healthy donors before blood collection. Briefly, $50-100 \mu \mathrm{L}$ capillary blood from donor fingers was sampled by lancets (Owen Mumford Ltd., Marietta, GA) according to the manufacturer manual, and collected into blood collection tubes pre-coated with EDTA (BD Biosciences, San Jose, CA). Blood was diluted with phosphate buffered saline (PBS) solution at a 1:1 ratio. Diluted blood was stained with Hoechst $33342(0.1 \%, v / v$; Life Technologies, Grand Island, NY), calcein AM $\left(1 \mu \mathrm{g} \mathrm{mL}^{-1}\right)$, fluorescein isothiocyanate (FITC)-labeled antibody specific to human CD14 (15 $\mu \mathrm{g} \mathrm{mL}^{-1}$; Life Technologies, Carlsbad, CA), phycoerythrin (PE)labeled antibody specific to human CD15 (10 $\mu \mathrm{g} \mathrm{mL}^{-1}$; BioLegend, San Diego, CA), or Alexa Fluor (AF) 647-labeled antibody specific to human CD3 (10 $\mu \mathrm{g} \mathrm{mL}{ }^{-1}$; BioLegend), for $5 \mathrm{~min}$ at room temperature before being assayed. Blood samples were vortexed to avoid sedimentation before being used within $4 \mathrm{hr}$ of blood collection.

2.3. Surface treatment and centrifugation operation. The CMC channel surface was passivated with one of the following blocking solutions for $30 \mathrm{~min}$ at room temperature before it was completely air-dried: fetal bovine serum (FBS; Thermo Fisher Scientific), trypsin ( $0.25 \%$ or 
$2.5 \mathrm{~g} \mathrm{~L}^{-1}$ in EDTA; Thermo Fisher Scientific), bovine serum albumin with Tween-20 (BSA-T20, 10\% BSA and 1\% (v/v) T20 in PBS; Sigma-Aldrich, St. Louis, MO), Pluronic F127 (50\% in PBS; Sigma-Aldrich), polyethylene glycol (PEG, molecular weight of 8,000, 50\% in PBS; SigmaAldrich), Teflon (DuPont, Wilmington, DE), and poly-L-lysine conjugated with PEG (PLL-PEG, $0.1 \mathrm{mg} \mathrm{mL}^{-1}$; Susos AG, Dübendorf, Switzerland). After surface passivation, the CMC was filled with blood and Ficoll solution (1.077 $\mathrm{g} \mathrm{mL}^{-1}$; GE Healthcare Bio-Sciences, Pittsburgh, PA) before it was placed inside a $50 \mathrm{~mL}$ centrifuge tube that was partially filled with foam to stabilize the $\mathrm{CMC}$ under centrifugation. The centrifuge tube was spun in a benchtop centrifuge (Eppendorf, Hauppauge, NY) with brakes turned off to minimize liquid agitation in the CMC. To examine cell adhesion in the CMC, motions of RBCs and WBCs under flow were induced by slightly tapping tubing connection to the $\mathrm{CMC}$ while observing cells in the $\mathrm{CMC}$ under fluorescence microscopy.

As a reference cell sorting technique, $100 \mu \mathrm{L}$ blood layered on top of $100 \mu \mathrm{L}$ Ficoll solution in a $200 \mu \mathrm{L}$ vial was spun at $400 \mathrm{~g}$ for $30 \mathrm{~min}$ as advised by the manufacturer instruction. To examine viability of unprocessed blood cells, $2 \mu \mathrm{L}$ intact whole blood was covered by a glass slide before being scanned and imaged.

All centrifugation experiments were conducted at room temperature.

2.4. Imaging and analysis. Blood separation was recorded using an imaging setup comprising an inverted microscope (Benz Microscope Optics Center, Ann Arbor, MI) and a digital color camera (BigCatchUSA, Torrance, CA). Photo collages of entire channels were stitched from individual images or video frames. Bright field and fluorescence images showing fluorescently labeled blood cells were recorded using a fluorescence microscope (AXIO Observer.Z1) attached 
with a CCD camera (AxioCam MRm), before the images were processed and stitched into a mosaic by software AxioVision (all from Carl Zeiss Microscopy, LLC, Thornwood, NY).

We used fluorescence signals for identifying blood cells. Specifically, cells without any staining indicated RBCs, whereas cells with Hoechst staining indicated WBCs. Since populations of eosinophils (CD193+) and basophils (CD123+) are relatively small compared with neutrophil population, CD15+ and Hoechst-stained blood cells were referred to directly as PMNs. PBMC population was estimated by differential counts between WBCs and PMNs. Platelets were ruled out during cell enumeration based on their small size relative to typical WBCs.

Blood cell counts were analyzed along the CMC's vertical main branch. The channel distance, $d$, was defined as the length measured from the bottom of the main branch. In normal situations, RBCs would sediment to the bottom of the main branch after centrifugation of the CMC. Formation of RBC sediments prohibited WBC enumeration within the pellet; therefore, cell counts started at different distances, depending on pellet size. We arbitrarily set an "infinite" value for $\mathrm{RBC}$ counts to show a steep increment in $\mathrm{RBC}$ numbers, indicating $\mathrm{RBC}$ pellet when accumulation started to appear. Recovery rate of PBMCs at any given position along the CMC main branch was defined as the PBMC count above the position divided by all PBMCs counted in the main branch. Purity rate of PBMCs was defined as the PBMC count above a position along the CMC main branch divided by the sum of PBMCs and PMNs above the position. Three additional terms were further quantified to examine immune cell sorting in the CMC (Fig. S1). PMN spread distance measured the distance for $70 \%$ of all PMNs from the bottom of the main channel. PBMC spread distance measured the distance for $70 \%$ of all PBMCs from the top 
position of PBMC distributions. PMN-PBMC separation measured the distance between the two regions defined by PMN spread distance and PBMC spread distance.

Statistical analysis was conducted with $n \geq 3$ independent experiments. Error bars represent one standard deviation (s.d.).

\section{RESULTS AND DISCUSSION}

\subsection{Design and operation of the CMC}

Leveraging the intrinsic optical transparency of PDMS and the capability to create microscale features in PDMS using soft lithography, herein we developed a simple biomedical device termed Centrifugal Microfluidic Chip (CMC) to integrate blood cell separation and in situ cellular analysis and enumeration. Using PDMS and glass as building materials, the CMC was optically transparent with low autofluorescence (Fig. S2), beneficial for fluorescence-based cellular analysis and enumeration.

The CMC was a single-layer microfluidic chip containing a simple channel network. Specifically, to facilitate fluid exchange and sample recovery, the CMC contained a $2.5 \mathrm{~cm}$ long, vertical main branch, a middle side branch connected to the center of the main branch, and a bottom side branch connected to the main branch bottom (Fig. 2a; please see Fig. $\mathbf{S 3}$ for detailed description of the CMC dimensions). Design of the CMC had several unique features in relation to its functionalities. The CMC main branch was a long straight channel built with location markers for easy alignment during imaging and analysis. The CMC main branch was $0.5 \mathrm{~mm}$ wide, and thus it was within the field of view (FOV) for a single linear scanning using a $10 \times$ objective in typical microscopes. In conjunction to a thin $\mathrm{CMC}$ channel, using $10 \times$ objectives also provided a large depth of focus (DOF) to image cells in the main branch, without missing 
cells due to out-of-focus or a need for resorting multiple scanning at different focal planes. Besides the main branch, the middle and bottom side branches allowed for extracting PBMCs and PMNs for subsequent examination after cell sorting. These two side branches were narrower (0.1 mm wide) than the main branch to minimize fluid mixing with the main branch during CMC operation. In addition, to minimize contamination at the blood-DGM interface during liquid exchange and centrifugation [16], two micropost barrier arrays were placed inside the main branch on top and below the middle side branch to stabilize flow (Fig. 2a and Fig. S3). Inlet and outlet holes of the CMC allowed operators to conveniently load solutions using pipettors or syringes.

The entire surface of the CMC was first passivated with a blocking solution to prevent non-specific adhesion of blood cells (Fig. 2b i\&ii). After the CMC was air-dried (Fig. 2b iii), about $0.25 \mu \mathrm{L}$ blood (diluted in PBS with a 1:1 $v / v$ ratio) was injected into the CMC main branch through the main branch inlet, and stopped at the barrier array (Fig. 2b iv). About $1 \mu \mathrm{L}$ Ficoll solution was then injected into the CMC main branch through the middle side branch inlet and exited via the bottom side branch outlet (Fig. 2b v\&vi). Thus, the upper portion of the main branch above the middle side branch (about $1 \mathrm{~cm}$ long) contained blood samples to be processed, while the bottom portion of the main branch below the middle side branch (about $1.5 \mathrm{~cm}$ long) contained the Ficoll solution (Fig. S3). The CMC was then packaged into a $50 \mathrm{~mL}$ centrifuge tube embedded with a foam stabilizer before centrifugation using a benchtop centrifuge (Fig. 2c). After centrifugation, immune cells in the blood redistributed along the CMC main branch according to their relative densities (Fig. 2d). Owing to its simple design and error-proof operation, the CMC could be operated without using a microscope. Blood cell sorting in the CMC main branch was analyzed immediately after centrifugation to minimize blood evaporation. 


\subsection{Surface passivation}

As PDMS has a dangling molecular structure and dynamic surface properties, a list of potential blockers [17-21] was examined for surface passivation of PDMS surfaces in the CMC to prevent blood cell adhesion (Supplementary Table 1). We used no-glass CMCs for surface passivation tests because of the ease to fabricate them. The majority of blockers examined in this work, including FBS, BSA-T20, Pluronic F127, and Teflon, did not lead to successful blood cell sorting in the $\mathrm{CMC}$ after centrifugation at 1,000 $\mathrm{g}$ for $10 \mathrm{~min}$. Specifically which blockers were involved in different operation failures were listed in Supplementary Table 1. Besides blood cells adhered to the CMC main branch surface (Fig. 3a i), cell agglutination as large clumps occurred at different main branch locations (Fig. 3a ii). As air bubbles could inevitably form during sample loading, these bubbles had led to cavitation in the main branch (Fig. 3a iii). It was difficult to completely remove highly hydrophobic Teflon solution from hydrophobic PDMS channels. Teflon residue could lead to the formation of multiphase bubbles and droplets (Fig. 3a iv). Due to an arching effect, the micropost barrier array designed for stabilizing flow became a physical obstacle to resist movement of cells and air bubbles (Fig. 3a v). Loosely packed RBC patches were also observed along the main branch, which became hurdles to RBC sedimentation (Fig. 3a vi).

Analyses of spatial distributions of blood cells along the main branch for CMCs treated with BSA and Pluronic F127 showed that both RBCs and WBCs remained widely spread out along the entire CMC main branch (Fig. 3b). We further examined which types of blood cells were adherent to PDMS surfaces in the CMC, with results showing that RBCs, but not WBCs, adhered to PDMS surfaces blocked with BSA-T20, and Pluronic F127 (Fig. 3c). 
Three blockers were identified in this work capable of passivating PDMS surfaces in the CMC. When the CMC was passivated with PEG, PLL-PEG, or trypsin, blood separation after centrifugation at $1,000 \mathrm{~g}$ for $10 \mathrm{~min}$ resembled to what is typically seen in conventional large centrifugation tubes (Fig. 4a). Both PEG and PLL-PEG have been commonly used as blockers, given the multivalent hydrophobic interactions from its repeating methylene units in PEG [22, 23], and the polycationic PEG grafted copolymer with a PLL backbone which strongly adsorbs onto negatively charged surfaces in aqueous solution [24]. Detailed explanation as how exactly trypsin, which is commonly used for detaching adherent cells, interact with PDMS to prevent blood cell adhesion will require an in-depth investigation and is beyond the scope of this work. When centrifugation was increased from $10 \mathrm{~min}$ to $20 \mathrm{~min}$ and $30 \mathrm{~min}$ at 1,000 $\mathrm{g}$ for the CMCs treated with PEG, PLL-PEG, and trypsin, more RBCs sank to the main branch bottom (Fig. 4b). The counts of contaminant RBCs above RBC pellets were plotted in Fig. 4c, which showed reduced $\mathrm{RBC}$ contaminant counts as the $\mathrm{CMC}$ were spun longer. Trypsin-coated CMCs not only had the least RBC contaminant counts even after 10 min spin, but also had RBC contaminant counts sharply dropped to only a few for a total of $20 \mathrm{~min}$ spin. Although the countable WBCs above RBC pellets dropped as spin time increased (Fig. 4d), PMNs were separated further from PBMCs and immersed in RBC pellets, thus leading to improved separation of both WBC subpopulations. The final amounts of WBCs after $30 \mathrm{~min}$ spin of CMCs treated with PEG, PLL-PEG, and trypsin were almost the same, suggesting an equilibrium amount of PBMCs has reached. Quantitative analysis of spatial distributions of RBCs and WBCs clearly revealed their dynamics in the CMC main branch (Fig. 4e). Most RBCs sank and formed dense pellets at the main branch bottom after the first $10 \mathrm{~min}$ of centrifugation with PLLPEG and trypsin passivation, whereas RBCs still scattered over the entire main branch with PEG 
passivation. Moreover, two WBC regions, one residing on top of RBC pellets and another residing near the micropost area, emerged after $30 \mathrm{~min}$ spin, resembling the typical layer distribution in conventional centrifugal tubes. Therefore, both PLL-PEG and trypsin had a comparable surface passivation effect and were better blockers than PEG for the CMC. We further validated that neither RBCs nor WBCs adhered to PDMS surfaces of the CMC treated with PEG, PLL-PEG, or trypsin (Fig. 4f).

For the remaining study, we chose to use trypsin as the blocker for surface passivation of the CMC, as it is a common biological solution, and notably, significantly smaller populations of RBCs were left above the RBC pellet region even after a 10 min centrifugation, clearly demonstrating well sorted and enriched immune cell subpopulations. Moreover, PLL-PEG is a costly synthetic chemical, limiting its accessibility to most biological laboratories.

\subsection{Blood centrifugation and cell distribution}

Simple fluid mechanics calculations revealed that the maximum rotational acceleration used in this study, 1,500 g, could exert a pressure as high as 55 psi to the CMC. Contrary to centrifugal apparatus made of hard plastics and glass, the structural material of the CMC, PDMS, was soft enough to deform and distort significantly at such a high spin speed [25, 26]. Although blood components could separate and distribute quickly during centrifugation, resilient PDMS channels experienced significant volume and shape change when the CMC returned to a stationary state, which would substantially and adversely distort blood cell distribution, leading to severe crosscontamination among separated blood cell layers.

To investigate how the structural and mechanical factors of the CMC would play a role in affecting its blood cell sorting performance, we systematically modulated the extent of PDMS 
channel deformation during centrifugation by bounding glass slides tightly against the $2 \mathrm{~mm}$ thick PDMS channel layer. Three CMC configurations, termed no-glass, bottom-glass, and glass-sandwich, were assembled, with no-glass CMC without any bounded glass slide, bottomglass $\mathrm{CMC}$ with a glass slide bounded to the $\mathrm{CMC}$ bottom surface, and glass-sandwich CMC with two glass slides bounded to the top and bottom surfaces of the CMC (Fig. 5a). After centrifugation at 1,000 $\mathrm{g}$ for $10 \mathrm{~min}, \mathrm{PBMCs}$ became separated from PMNs in all configurations (Fig. 5b). We further analyzed the quality of cell separation by PMN and PBMC spread and PMN-PBMC separation distance (see Methods for their definitions; Fig. S1). While there was no apparent difference in PMN spread distance among different configurations (Fig. 5c), providing more structural supports for CMC showed a clear trend of reducing PBMC spread distance (Fig. 5d). With consistently the farthest separation between PMN and PBMC populations (Fig. 5e\&f), the glass-sandwich configuration led to the best PBMC purity from CMC operations. Together, these observations supported that bounding the $\mathrm{CMC}$ with rigid glass slides had reduced PDMS channel deformation during high-speed centrifugation and consequently reduced cell redistribution and cross-contamination [25, 26].

Since RBCs can easily be removed from blood samples by RBC lysis buffer, herein we focused on analyzing spatial distributions of WBCs in the CMC after centrifugation at 1,000 g for 10 min. Taking PBMCs as target cells and PMNs as contaminants, recovery and purity rates of PBMCs were analyzed as a function of channel distance $d$ in no-glass, bottom-glass, and glass-sandwich CMCs (see Methods for definitions of recovery and purity rates; Fig. 5g\&h), with results showing that in all three device types, PBMC recovery and purity rates decreased and increased with channel distance $d$, respectively. The glass-sandwich CMC showed the best PBMC recovery performance, as it allowed collection of more PBMCs at a shorter distance from 
the main channel top. On the other hand, both bottom-glass and glass-sandwich CMCs showed better sorting performance for PBMCs than no-glass CMCs, owing to purer PBMC populations at a shorter distance from the main channel top. To further evaluate their trade-off, PBMC purity rate was correlated with its recovery rate (Fig. 5i). A data point closer to the top-right corner in such a correlation plot would mean a desirable condition to attain concurrent high recovery and purity values. Thus, Fig. 5i revealed that centrifugation of the glass-sandwich CMC could achieve both recovery and purity rates of above $90 \%$ for PBMCs after centrifugation at 1,000 g for $10 \mathrm{~min}$. Based on the recovery-purity correlation curve, generally speaking, no-glass CMCs were the worst and glass-sandwich CMCs were the best for attaining concurrent high recovery and purity values for PBMCs.

We next examined how different centrifugation acceleration rates $(500 \mathrm{~g}, 1,000 \mathrm{~g}$, and $1,500 \mathrm{~g}$ ) could affect blood cell separation in the CMC. To this end, we chose to use glasssandwich CMCs and further reduce the PDMS channel-layer thickness to about $400 \mu \mathrm{m}$, such that the effect of glass slides as structural supports for the CMC became more prominent. Compared with 500 and 1,500 g spin speeds, 1,000 g acceleration consistently showed the least PMN (Fig. 6a) and PBMC (Fig. 6b) spread distances and the farthest separation between the two cell populations (Fig. 6c), allowing better PBMC sorting with concurrent high recovery and purity values. On the other hand, in all experiments except those with $500 \mathrm{~g}$ acceleration, peaks of PBMCs and PMNs were well separated from each other along the main branch of the CMC after 10 min of centrifugation (Fig. 6d). The cell distribution plot also showed that centrifugation at 1,000 $\mathrm{g}$ yielded the highest number of PBMCs and minimum overlap between PMN and PBMC populations compared with the other two accelerations (Fig. 6d). While varying the acceleration condition had little effect on PBMC recovery rate (Fig. 6e), it did show 
significantly improved PBMC purity rate from $500 \mathrm{~g}$ to $1,000 \mathrm{~g}$ but compromised purity rates at $1,500 \mathrm{~g}$ (Fig. 6f). The recovery-purity correlation clearly showed a wide range of recovery rates or high purity rates closed to $100 \%$ at 1,000 g (Fig. 6g). In particular, a purity of $97 \%$ and recovery of $95 \%$ could be achieved at 1,000 $\mathrm{g}$ for PBMCs. Interestingly, centrifugation of the CMC at 1,500 g did not further improve separation between PBMCs and PMNs when compared with centrifugation at $1,000 \mathrm{~g}$; this observation was further confirmed when the recovery and purity rates of PBMCs were analyzed under different centrifugation acceleration rates (Fig. 6e-g). We suspect that centrifugation of the CMC at 1,500 $\mathrm{g}$ might be too forceful to cause unexpected cell dynamic behaviors or too much material deformation in the CMC as investigated in Fig. 5, leading to compromised cell sorting. All together, an acceleration of 1,000 g for CMC operation was the optimal condition for isolating PBMCs from blood.

\subsection{Blood cell health, CMC post-spin stability and multi-color imaging}

From a design perspective, although the thin CMC channel allowed for single scanning under the limited DOF (see Design and operation of the CMC for detailed discussion), it created a larger shear stress when cells migrated in the channel. To validate any adverse effects that might incur to cells during cell sorting using the CMC, we compared cell viability using cells undergone a vial-scale Ficoll centrifugation and cells from intact whole blood. Using Hoechst and a viability marker calcein AM, WBCs could be identified live or dead according to the calcein AM fluorescence (Fig. 7a). Regardless CMC or vial operations, cell viability was almost 100\% within 40 min after spinning (Fig. 7b). Since Ficoll solution is not a cell medium, unhealthy cells emerged after $1 \mathrm{hr}$ of culture in the Ficoll solution. The number of unhealthy cells using CMC operations was less than that of the vial method, possibly attributable to shorter Ficoll 
exposure and spinning times in CMC operations (10 min for CMC vs. $30 \mathrm{~min}$ in the vial method). In all cases, cell viability was above $97 \%$ within $1.5 \mathrm{hr}$ after centrifugation processes or within 4 hr after blood collection without centrifugation. Such high cell viability rates confirmed a minimum cell damage by CMC operations.

Besides separating and extracting cells, continuous scanning of in situ assays is also an important consideration for CMCs. To verify the stability for short-term handling and storage, CMCs were repeatedly operated, scanned, and stored. Using Hoechst-stained blood, CMC main channels were imaged 4 times (Fig. 7c). Formation of RBC pellets was stable and did not scatter towards the channel top even though the chips were laid horizontally and moved around places. However, an air-liquid interface formed and steadily grew towards the channel bottom due to evaporation. With the perfectly collapsed Hoechst-stained cell distribution curves, neither a global shift nor a redistribution of the cell location was observed, confirming such a moving interface had negligible effect on cell sorting within $1.5 \mathrm{hr}$ after centrifugation (Fig. 7d). Therefore, short-term in situ examination of evolving cell behaviors or dynamics is feasible. Multi-color imaging is another critical aspect for the delivery of a versatile CMC platform capable of comprehensive cellular characterization $[11,12]$. Besides CD15 antibody, antibodies for CD14 and CD3 were added to blood before centrifugation. Cells stained specifically against these antibody markers, with CD15+ PMNs below microposts and CD14+ monocytes or CD3+ T cells above microposts, were detected (Fig. 7e). Since cells retained fluorescence signals on cell membrane, these results indicated that cells undergone CMC operations maintained good cell surface integrity. As these three major important immune cell subpopulations are related to diverse diseases, and a large pool of antibody markers to identify hematopoietic stem cells (HSCs) or CD4 and CD8 T cell subpopulations are available, CMCs 
can be further developed into a blood diagnostic device or a CBC platform for blood examination or cyto-informatics, respectively, in healthcare or critical care applications.

It would be ideal to achieve baseline separation between PMNs and PBMCs by centrifugation of the CMC. However, as the densities of PMNs and PBMCs overlap with each other (Fig. 1a), complete separation between PBMCs and PMNs is, in principle, not possible by centrifugation. In addition, operations to load solutions into the CMC might cause contaminations of the DGM and blood samples at their interface, further compromising separation efficiency of the CMC for isolating PBMCs from other blood cells including PMNs. Lastly, the cell distribution was the same with or without the barrier array. Although the microposts are optional components, and removing them can avoid channel clogging, we found them useful when monitoring solution exchange without using a microscope.

\section{CONCLUSION}

In this work, we developed a centrifugal microfluidic device termed the $\mathrm{CMC}$ as a promising, portable microfluidic platform for rapid and efficient blood cell sorting and cellular imaging and analysis. The CMC, based on a 1-layer microfluidic design, could achieve immune cell sorting and enumeration by one single centrifugation step using common technical periphery available in a biomedical laboratory. We further identified different surface passivation methods suitable for the CMC to prevent non-specific binding of blood cells to PDMS surfaces; this is important as blood separation in confining microfluidic environments is very sensitive to cell-surface interactions due to a high surface-to-volume ratio in microfluidics. We also devised and validated a glass-sandwich channel-layer assembly to strengthen the CMC mechanical property to minimize PDMS channel deformation during high-speed centrifugation. A single step 
centrifugation of the $\mathrm{CMC}$ for 10 min could achieve both recovery and purity rates $>95 \%$ for PBMCs, a WBC viability rate $>98 \%$, and stable separation within the channel from minimally processed whole blood. Importantly, we further demonstrated 4-color immunostaining and fluorescence microscopy for in situ cellular analysis in the $\mathrm{CMC}$, further supporting the CMC as a versatile platform for blood-based diagnosis and disease monitoring.

While there are numerous sophisticated microfluidic cell sorting and imaging platforms reported in the literature $[1,9,10,27-29]$, however, very few of them can be used directly for whole blood samples due to intricate design, bulky setup, and complicated control and operations. The CMC can process a few microliters of blood, reducing blood consumption to a thousandth of conventional methods, which is especially advantageous for pediatric patients. In this work, we have further devised an optimal device design and operation parameters to shorten the centrifugation time to $10 \mathrm{~min}$, shorter compared to $30 \mathrm{~min}$ as advised by the manufacturer instruction. The compact and low-cost CMC that can achieve efficient and rapid separation of immune cells from complex background of minimally processed whole blood for on-chip cytometric examination will hold promise for blood-based diagnosis and disease monitoring in POC applications [30]. We envision that our newly invented Vacuum-Pressure Accelerated Movement (V-PAM) technology [31] can be further incorporated into CMCs in the future for improved operations such as automatic fluid filling and transferring to an extraction or concentration chamber.

\section{ACKNOWLEDGEMENTS}

We acknowledge financial support from the National Science Foundation (CBET 1263889 and CMMI 1536087), the National Institutes of Health (R01 HL119542), and the Michigan Center 
for Integrative Research in Critical Care (M-CIRCC). The Lurie Nanofabrication Facility at the University of Michigan, a member of the National Nanotechnology Infrastructure Network (NNIN) funded by the National Science Foundation, is acknowledged for support in microfabrication. 


\section{REFERENCES}

[1] Z.T. Yu, K.M. Aw Yong, J. Fu, Microfluidic blood cell sorting: now and beyond, Small, 10(2014) 1687-703.

[2] L. Sompayrac, How the immune system works, 4th ed.: Wiley-Blackwell; 2012.

[3] K.M. Aw Yong, Z.T.F. Yu, K.H. Guan, J. Fu, Types of Clinical Samples and Cellular

Enrichment Strategies, in: J.C. Love (Ed.) Micro- and Nanosystems for Biotechnology, Wiley-

VCH Verlag GmbH \& Co. KGaA, Weinheim, Germany, 2016, pp. 1-28.

[4] A.A. Okoye, L.J. Picker, CD4(+) T-cell depletion in HIV infection: mechanisms of immunological failure, Immunol Rev, 254(2013) 54-64.

[5] T.H. Kim, H. Hwang, R. Gorkin, M. Madou, Y.K. Cho, Geometry effects on blood separation rate on a rotating disc, Sensor Actuat B-Chem, 178(2013) 648-55.

[6] D.J. Kinahan, S.M. Kearney, M.T. Glynn, J. Ducree, Spira mirabilis enhanced whole blood processing in a lab-on-a-disk, Sensor Actuat a-Phys, 215(2014) 71-6.

[7] J.M. Park, M.S. Kim, H.S. Moon, C.E. Yoo, D. Park, Y.J. Kim, et al., Fully automated circulating tumor cell isolation platform with large-volume capacity based on lab-on-a-disc, Anal Chem, 86(2014) 3735-42.

[8] H. Shiono, H.M. Chen, T. Okada, Y. Ito, Colony-forming cell assay for human hematopoietic progenitor cells harvested by a novel continuous-flow cell separation method, J Chromatogr A, 1151(2007) 153-7.

[9] O. Strohmeier, M. Keller, F. Schwemmer, S. Zehnle, D. Mark, F. von Stetten, et al., Centrifugal microfluidic platforms: advanced unit operations and applications, Chem Soc Rev, 44(2015) 6187-229.

[10] D.J. Kinahan, S.M. Kearney, N.A. Kilcawley, P.L. Early, M.T. Glynn, J. Ducree, DensityGradient Mediated Band Extraction of Leukocytes from Whole Blood Using CentrifugoPneumatic Siphon Valving on Centrifugal Microfluidic Discs, PLoS ONE, 11(2016).

[11] K.I. Kamei, M. Ohashi, E. Gschweng, Q. Ho, J. Suh, J.H. Tang, et al., Microfluidic image cytometry for quantitative single-cell profiling of human pluripotent stem cells in chemically defined conditions, Lab Chip, 10(2010) 1113-9.

[12] J. Sun, M.D. Masterman-Smith, N.A. Graham, J. Jiao, J. Mottahedeh, D.R. Laks, et al., A microfluidic platform for systems pathology: multiparameter single-cell signaling measurements of clinical brain tumor specimens, Cancer Res, 70(2010) 6128-38.

[13] Z.T. Yu, H. Guan, M.K. Cheung, W.M. McHugh, T.T. Cornell, T.P. Shanley, et al., Rapid, automated, parallel quantitative immunoassays using highly integrated microfluidics and AlphaLISA, Sci Rep, 5(2015) 11339.

[14] Z.T. Yu, K. Kamei, H. Takahashi, C.J. Shu, X. Wang, G.W. He, et al., Integrated microfluidic devices for combinatorial cell-based assays, Biomed Microdevices, 11(2009) 54755.

[15] K. Kamei, S. Guo, Z.T. Yu, H. Takahashi, E. Gschweng, C. Suh, et al., An integrated microfluidic culture device for quantitative analysis of human embryonic stem cells, Lab Chip, 9(2009) 555-63.

[16] T.J. Li, L.M. Zhang, K.M. Leung, J. Yang, Out-of-plane microvalves for whole blood separation on lab-on-a-CD, J Micromech Microeng, 20(2010).

[17] H. Vaisocherova, E. Brynda, J. Homola, Functionalizable low-fouling coatings for labelfree biosensing in complex biological media: advances and applications, Anal Bioanal Chem, 407(2015) 3927-53. 
[18] D. Kim, A.E. Herr, Protein immobilization techniques for microfluidic assays, Biomicrofluidics, 7(2013).

[19] J.W. Zhou, A.V. Ellis, N.H. Voelcker, Recent developments in PDMS surface modification for microfluidic devices, Electrophoresis, 31(2010) 2-16.

[20] J.W. Zhou, D.A. Khodakov, A.V. Ellis, N.H. Voelcker, Surface modification for PDMSbased microfluidic devices, Electrophoresis, 33(2012) 89-104.

[21] Q.L. Yu, Q.H. Wang, B.M. Li, Q.Y. Lin, Y.X. Duan, Technological Development of Antibody Immobilization for Optical Immunoassays: Progress and Prospects, Crit Rev Anal Chem, 45(2015) 62-75.

[22] G.C. Shao, J. Wang, Z.H. Li, L. Saraf, W.J. Wang, Y.H. Lin, Poly(dimethylsiloxane) microchip-based immunoassay with multiple reaction zones: Toward on-chip multiplex detection platform, Sensor Actuat B-Chem, 159(2011) 44-50.

[23] B. Liu, P.J. Huang, X. Zhang, F. Wang, R. Pautler, A.C. Ip, et al., Parts-per-million of polyethylene glycol as a non-interfering blocking agent for homogeneous biosensor development, Anal Chem, 85(2013) 10045-50.

[24] I. Wong, C.M. Ho, Surface molecular property modifications for poly(dimethylsiloxane) (PDMS) based microfluidic devices, Microfluid Nanofluidics, 7(2009) 291-306.

[25] Q. Chen, G. Li, Y. Nie, S.H. Yao, J.L. Zhao, Investigation and improvement of reversible microfluidic devices based on glass-PDMS-glass sandwich configuration, Microfluid Nanofluid, 16(2014) 83-90.

[26] D.W. Inglis, A method for reducing pressure-induced deformation in silicone microfluidics, Biomicrofluidics, 4(2010).

[27] N.T. Huang, W. Chen, B.R. Oh, T.T. Cornell, T.P. Shanley, J. Fu, et al., An integrated microfluidic platform for in situ cellular cytokine secretion immunophenotyping, Lab Chip, 12(2012) 4093-101.

[28] X. Li, W. Chen, G. Liu, W. Lu, J. Fu, Continuous-flow microfluidic blood cell sorting for unprocessed whole blood using surface-micromachined microfiltration membranes, Lab Chip, 14(2014) 2565-75.

[29] S. Wang, K. Liu, J. Liu, Z.T. Yu, X. Xu, L. Zhao, et al., Highly efficient capture of circulating tumor cells by using nanostructured silicon substrates with integrated chaotic micromixers, Angew Chem Int Ed Engl, 50(2011) 3084-8.

[30] M.N. Gulari, A. Tripathi, M. Ghannad-Rezaie, N. Chronis, An Optofluidic Lens Array Microchip for High Resolution Stereo Microscopy, Micromachines-Basel, 5(2014) 607-21. [31] Z.T. Yu, M.K. Cheung, S.X. Liu, J. Fu, Accelerated Biofluid Filling in Complex Microfluidic Networks by Vacuum-Pressure Accelerated Movement (V-PAM), Small, 12(2016) 4521-30.

[32] Y. Takamura, K. Idegami, M. Kogi, H. Takabayashi, Method for collecting nucleated red blood cells via density-gradient centrifugation utilizing changes in blood cell density in: USPTO (Ed.), Japan Advanced Institute Of Science And Technology, Kanazawa Medical University, \& Kanazawa Institute Of Technology, USA, 2013. 


\section{FIGURE CAPTIONS}

Figure 1. Blood cell densities and sorting using density gradient centrifugation. (a) Scheme showing density distributions of human blood cells [32]. (b) Movement of blood cells upon centrifugation for human blood placed on top of the Ficoll solution. White arrows indicate cell movement directions.

Figure 2. Blood cell sorting using density gradient centrifugation in a microfluidic device. (a) Photos of the centrifuge microfluidic chip (CMC) filled with dyes (red for blood and blue for the Ficoll solution) for visualization. An array of microposts inside the main branch served as barriers for stabilizing flow. Dashed lines highlight microposts. (b) Operation procedure of the CMC for sorting blood cells. Initially, the CMC was empty (i). After passivation with a blocking solution (dark grey) (ii), the CMC was air dried (iii) before loading with blood specimens (red) from the main branch down to the barrier array (iv) and the Ficoll solution (blue) from the side branch to fill the remaining channels (v and vi). (c) the CMC loaded with blood and the Ficoll solution was inserted into a centrifuge tube before spinning in a benchtop centrifuge machine. Foam was placed inside the centrifuge tube underneath the CMC to absorb uneven pressure under high spin-speed. (d) Cartoon showing blood cell distribution expected after separation in the CMC.

Figure 3. Improper blood sorting in the CMC. (a) Phenomena leading to blood cell separation failure in the CMC. Please refer to Fig. S4a for occurrence locations inside the main branch. (i) Blood cells became adherent. (ii) Blood cell agglutination. (iii) Air bubble generation, anchoring, and congestion. (iv) Multiphase formation between blood and Teflon 
residue inside a Teflon-treated main branch. (v) Blood and bubble clogging due to the arching effect at the barrier array. (vi) Loosely packed RBC patches scattered over the main branch. (b) Spatial distributions of RBCs and WBCs along the main branch after centrifugation. The CMC was passivated with either (i) BSA or (ii) F127 as indicated. (c) Time-lapse images showing motions of RBCs (black, under bright field) and Hoechst-stained WBCs (blue, under fluorescence) under flow. No-glass CMCs were centrifuged at an acceleration of 1,000 g for 10 min.

Figure 4. Blood cell sorting in the CMCs passivated with PEG, PLL-PEG, and trypsin. (a) Photo collages showing blood separation along the main branch of the no-glass CMC treated with either (i) PEG, (ii) PLL-PEG, or (iii) trypsin as indicated, after 10 min centrifugation. (b) Magnified views showing blood cell distribution along the main branch of the no-glass CMC treated with either (i) PEG, (ii) PLL-PEG, or (iii) trypsin, after centrifugation for 10, 20, or 30 min as indicated. Please refer to Fig. S4b for locations where the images were taken. (c\&d) $\mathrm{RBC}(\mathbf{c})$ and $\mathrm{WBC}(\mathbf{d})$ counts above RBC pellets as functions of spin time and blocker. (e) Spatial distributions of RBCs and WBCs along the main branch. Results were obtained from a and b. (f) Time-lapse images showing motions of RBCs (black, under bright field) and Hoechststained WBCs (blue, under fluorescence ) under flow. A centrifuge acceleration of 1,000 g was used.

\section{Figure 5. Blood cell sorting performance by the CMC with different structural supports.}

(a) Cross-sectional schemes of the CMC with no-glass, bottom-glass, and glass-sandwich configurations at rest or deformed states under spinning. (b) Merged bright-field and 
fluorescence images showing Hoechst (blue, for staining WBCs) and CD15 (orange, for staining PMNs) staining. Images were taken below, at, and above the barrier array along the CMC main branch as indicated. (c-e) PMN (c) and PBMC (d) spread, and PMN-PBMC separation (e) distances as functions of different structural supports. (f) Spatial distributions of PMNs and PBMCs along the main branch of the CMC with no-glass, bottom-glass, or glass-sandwich configuration as indicated. (g\&h) Recovery $(\mathbf{g})$ and purity rates (h) of PBMCs as a function of channel distance $d$. (i) Correlation of PBMC purity versus recovery rates. Two reference lines (grey) were drawn to represent recovery and purity rates at $90 \%$. CMCs were centrifuged at an acceleration of $1,000 \mathrm{~g}$ for $10 \mathrm{~min}$.

\section{Figure 6. Blood cell sorting performance of the CMC with glass-sandwich configuration} under different centrifugal accelerations. (a-c) PMN (a) and PBMC (b) spread, and PMNPBMC separation (c) distances as functions of different centrifugal accelerations. (d) Spatial distributions of PBMCs and PMNs along the CMC main branch after centrifugation at $500 \mathrm{~g}$, $1,000 \mathrm{~g}$, or 1,500 $\mathrm{g}$ for $10 \mathrm{~min}$ as indicated. (e\&f) Recovery rate (e) and purity rate (f) of PBMCs as functions of channel distance $d$. (g) Correlation of PBMC purity rate versus recovery rate. Two reference lines (grey) were drawn to represent recovery and purity rates at $90 \%$.

Figure 7. Validation of cell health, CMC operations and multi-color fluorescence imaging. (a) Bright field and fluorescence images of dead and live cells showing Hoechst (blue, for staining WBCs) and calcein AM (green, for staining live cells). (b) Comparison of live cell populations between the $\mathrm{CMC}$ and vial-based operations. A red region representing the range of live cell populations from intact whole blood was included for comparison. Vial centrifugation 
was performed at an acceleration of $400 \mathrm{~g}$ for $30 \mathrm{~min}$. (c) Changes of contents in CMCs at different time points. Hoechst staining (pseudo color: green) was imaged. (d) Redistributions of Hoechst-stained cell in c. (e) Identification of WBC subpopulations through Hoechst (blue), and antibodies against CD14 (green; a monocyte marker), CD15 (orange; a PMN marker), and CD3 (red; a T-cell marker). CMCs were centrifuged at an acceleration of 1,000 $\mathrm{g}$ for $10 \mathrm{~min}$. 


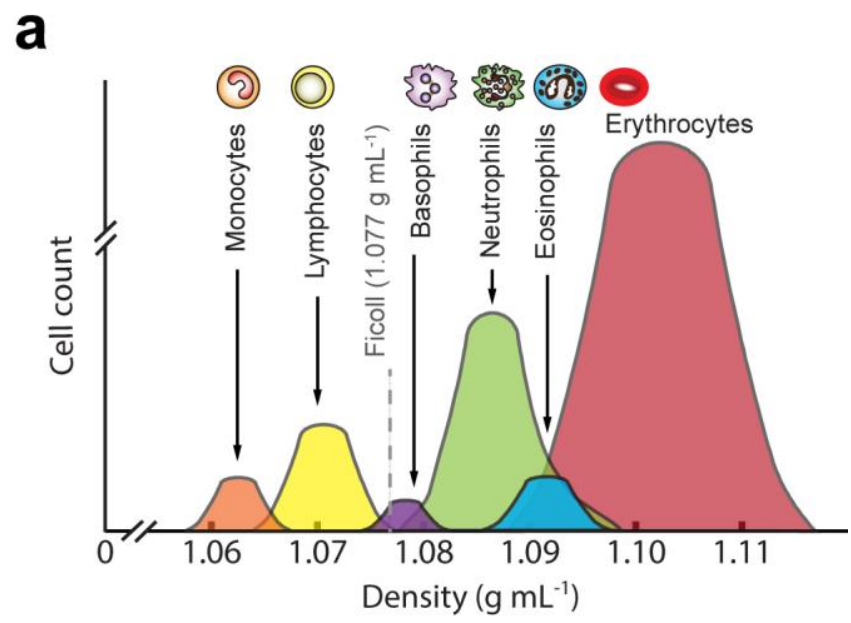

b

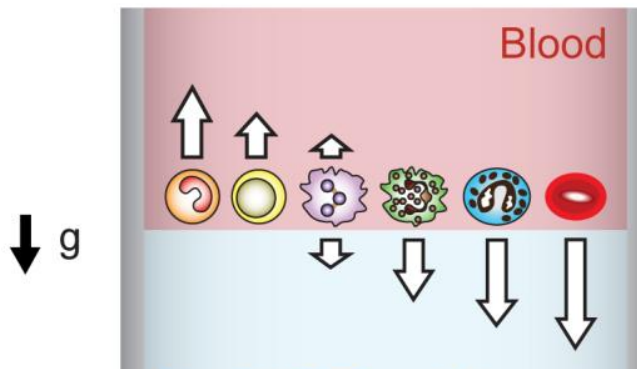

Ficoll (1.077 $\mathrm{g} \mathrm{mL}^{-1}$ ) 
a
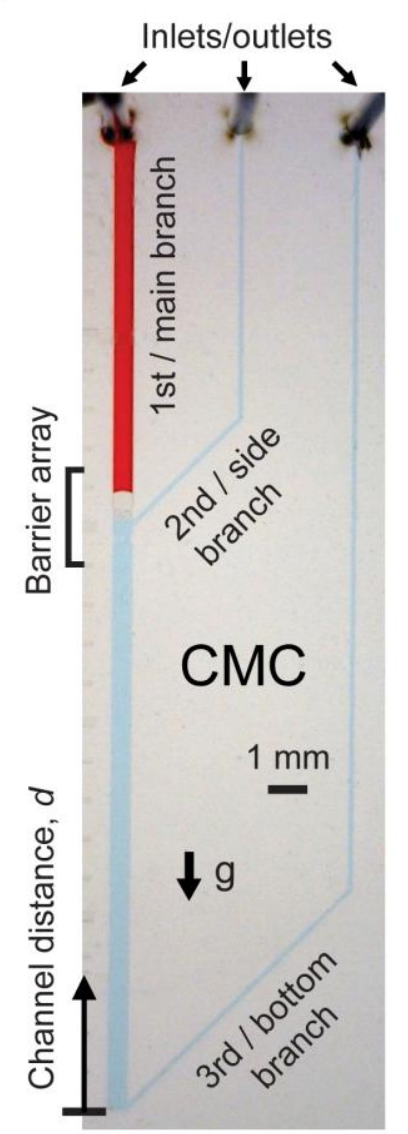

b
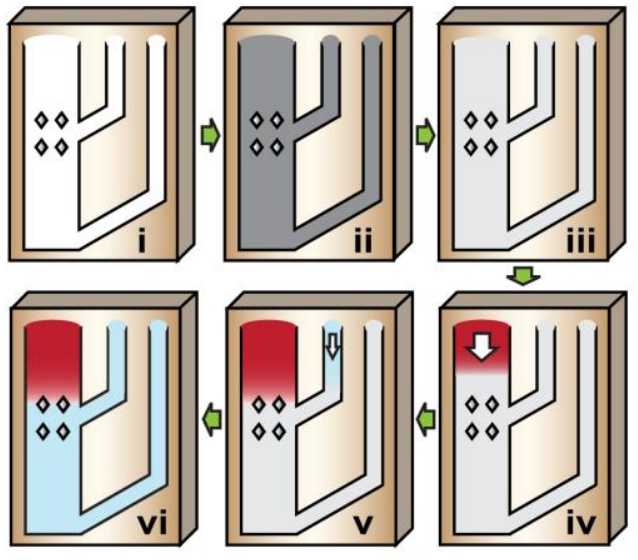

$\square$ Blocker $\square$ Passivation $\square$ Blood $\square$ DGM

c

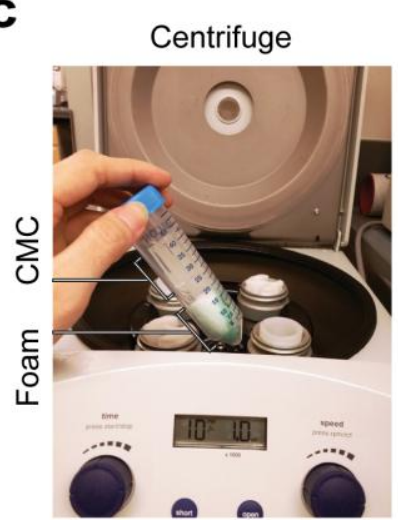

d

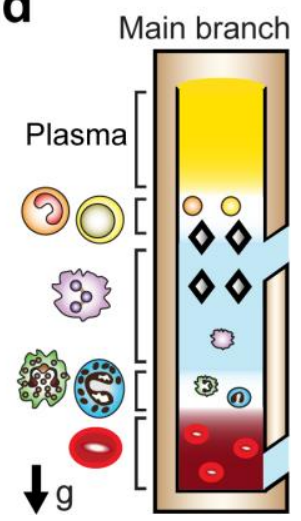


a
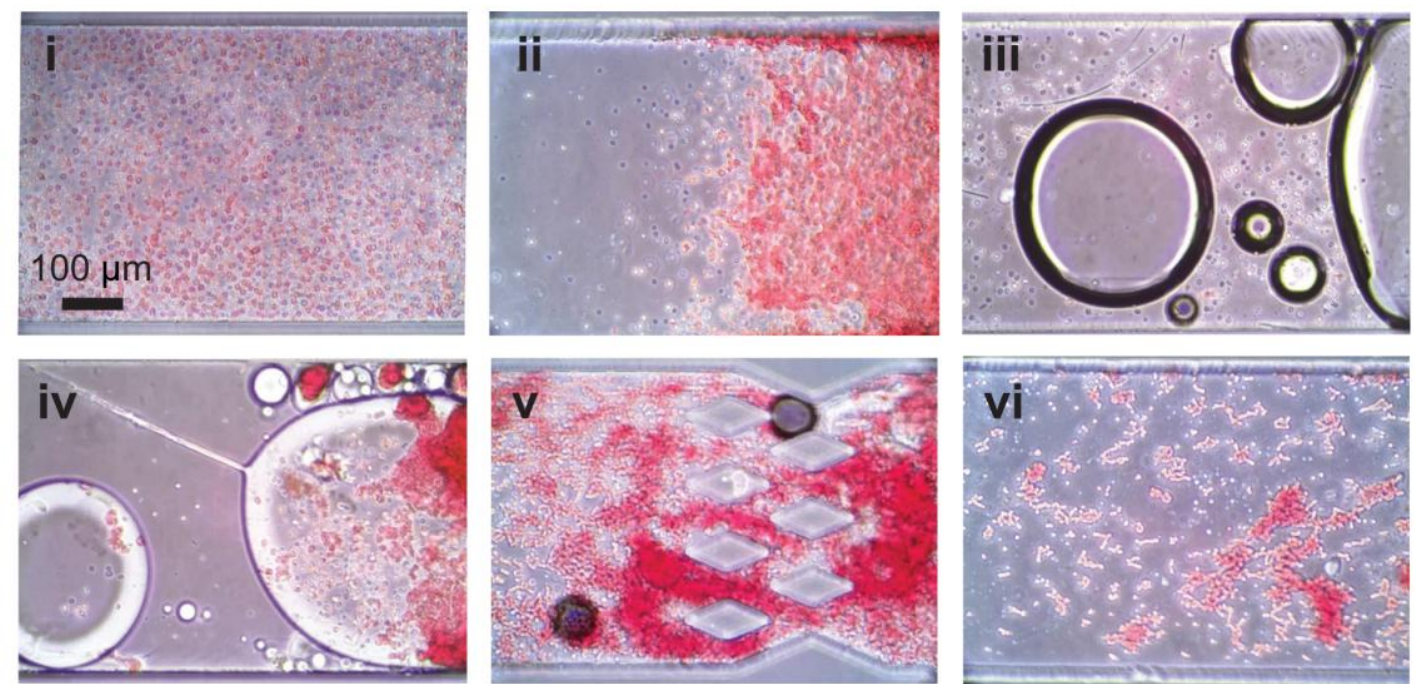

b

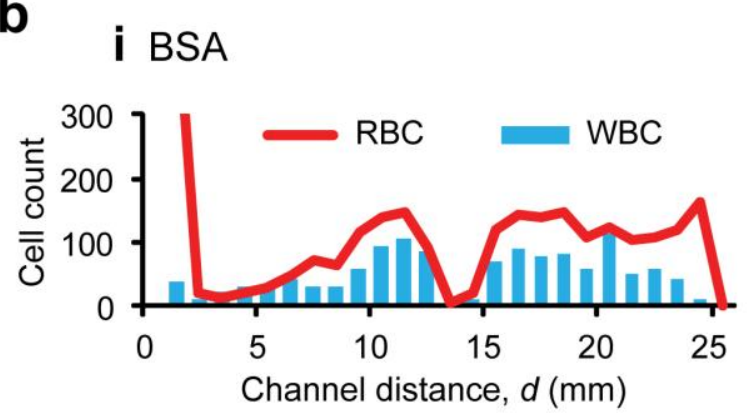

ii F127

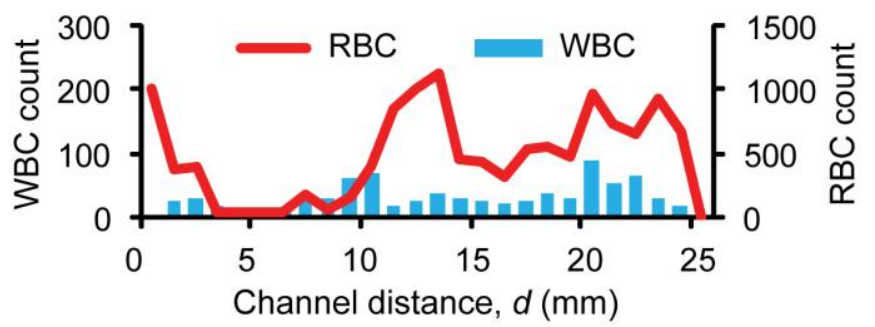

C
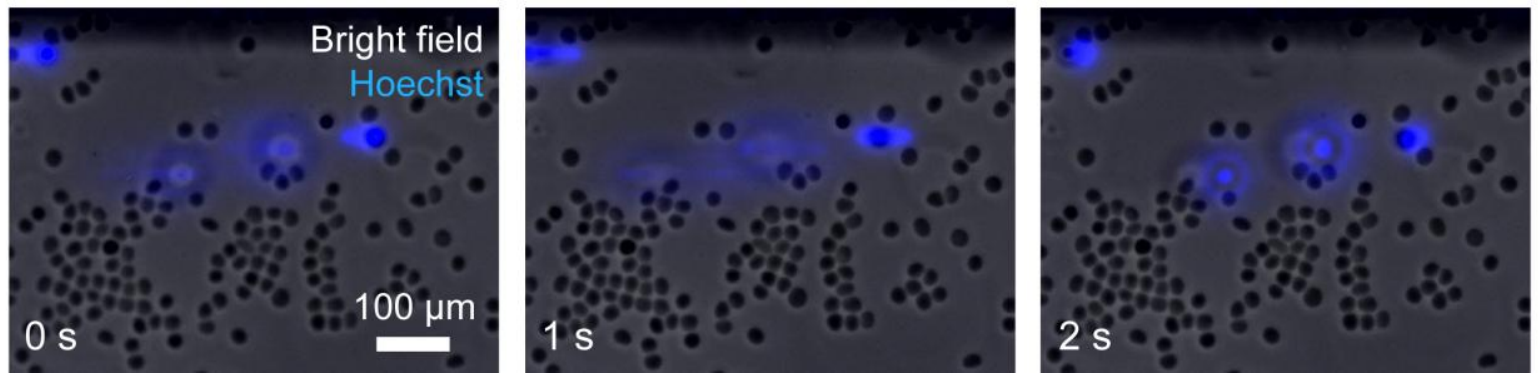
a

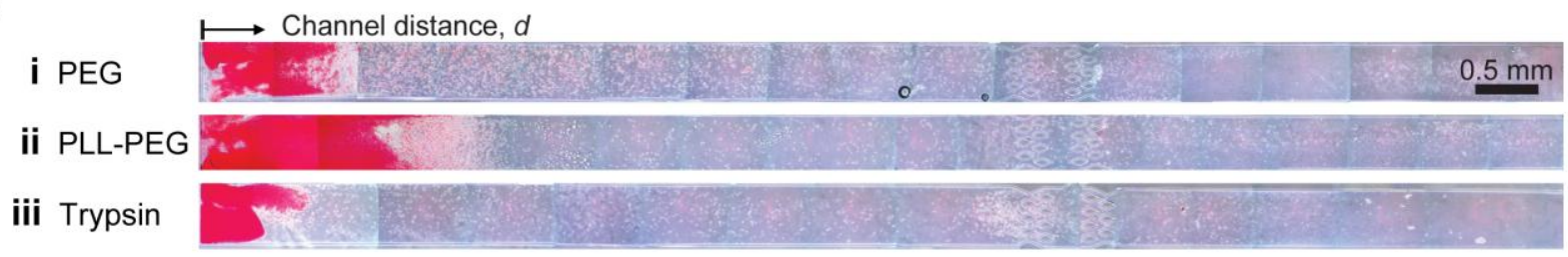

b
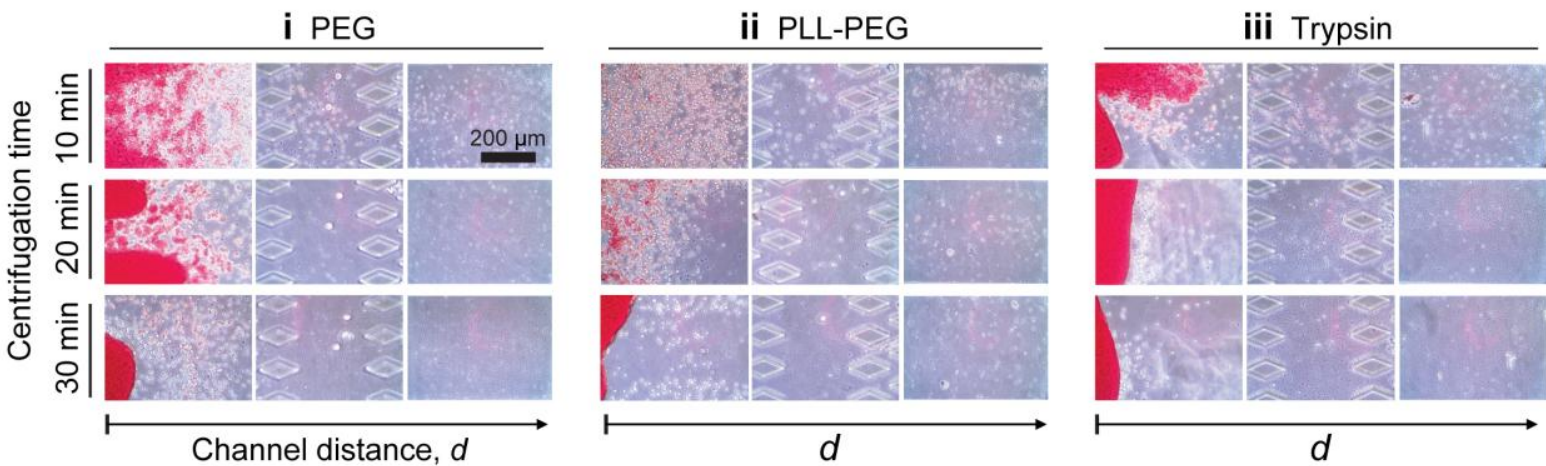

C

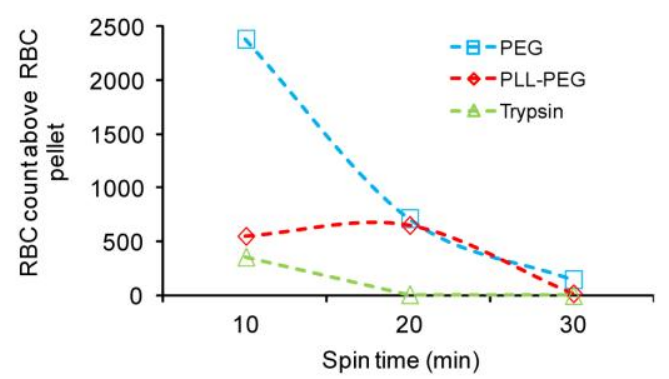

d

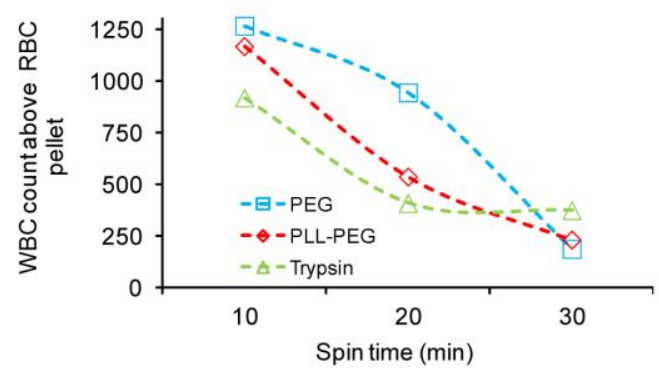

e
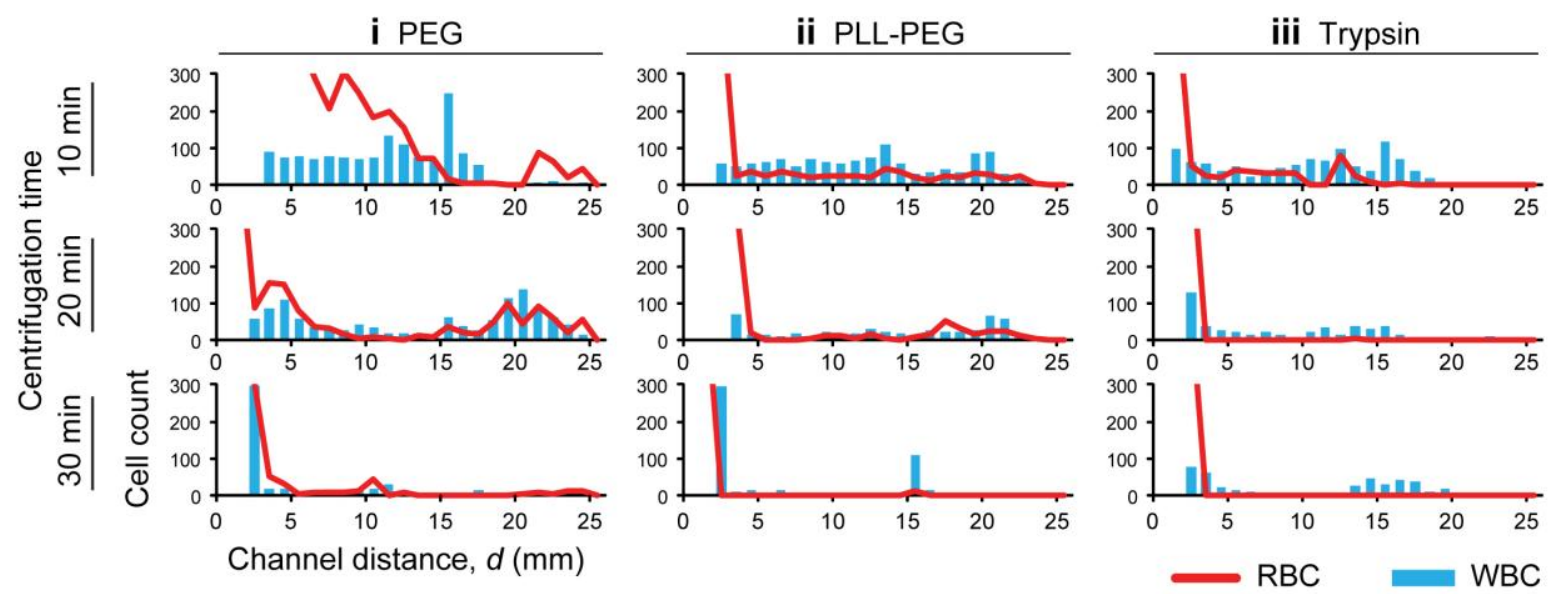

f
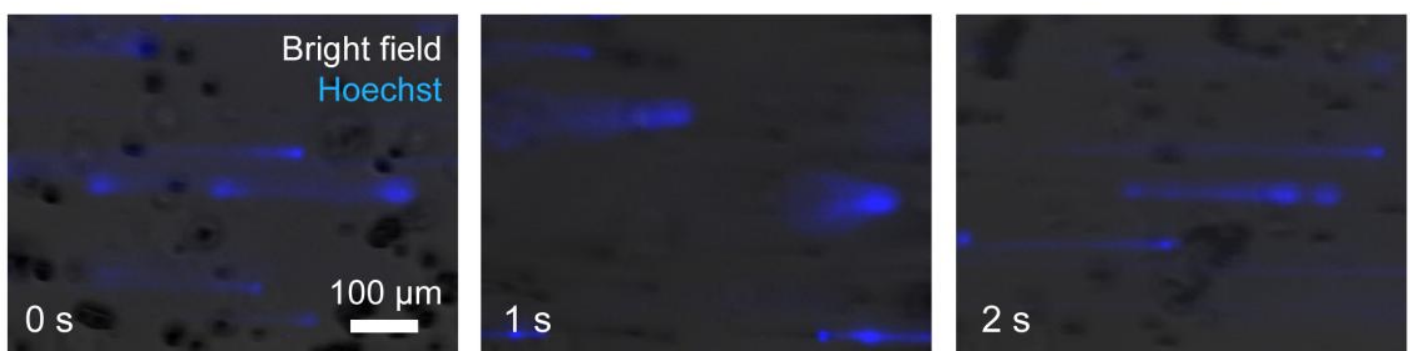
a
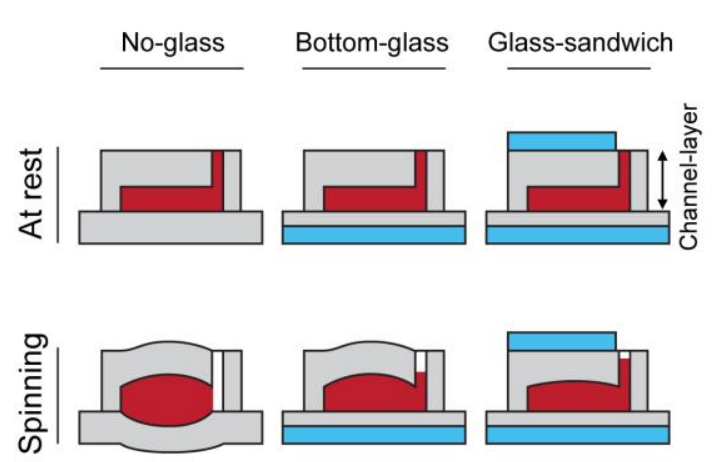

Blood

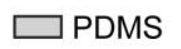

C

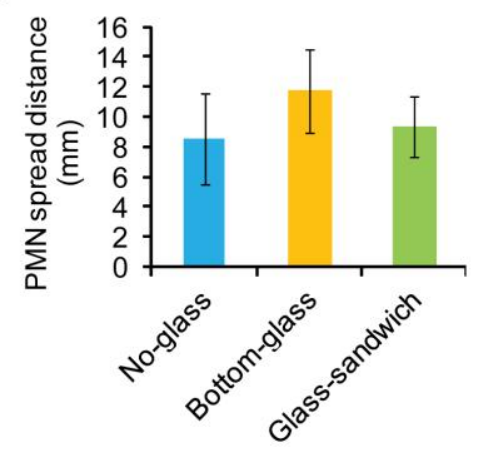

f

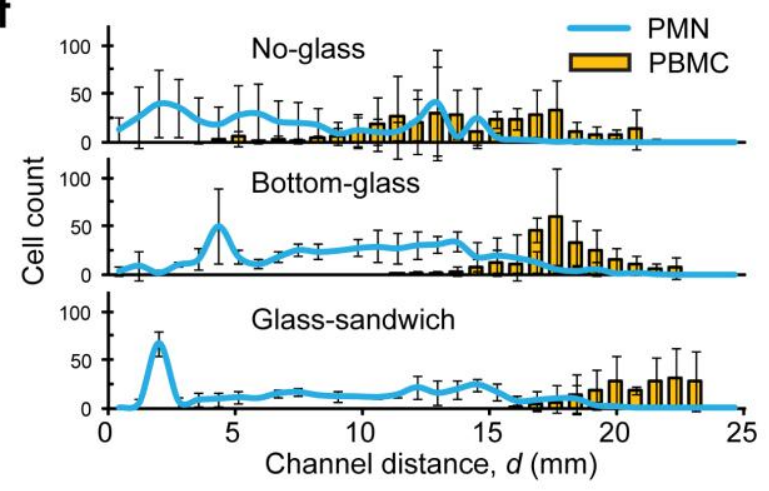

h

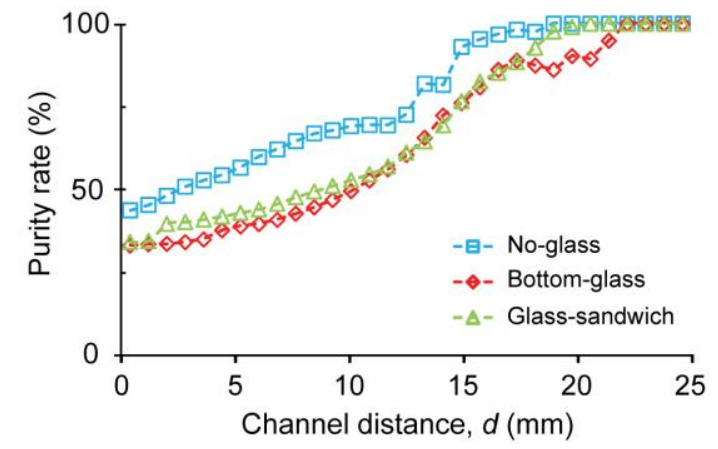

b

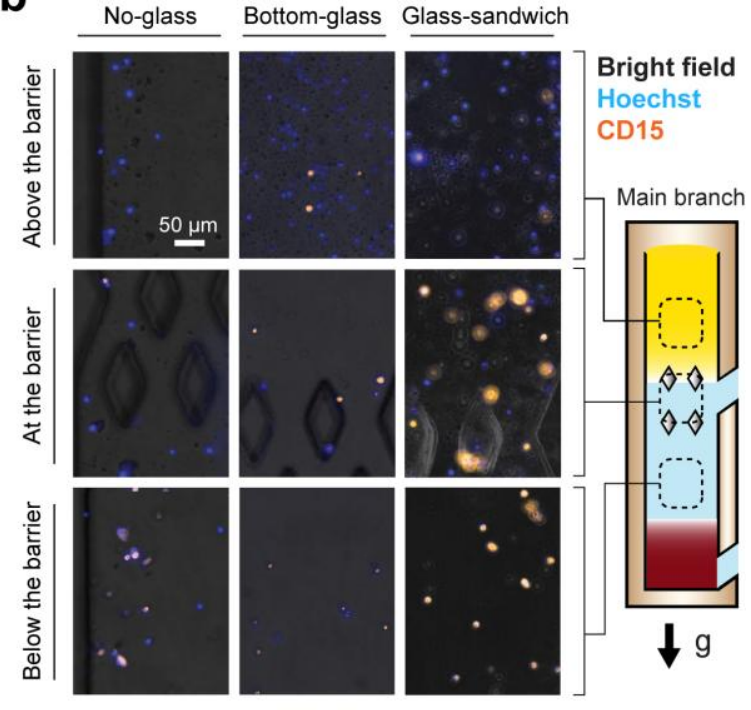

d

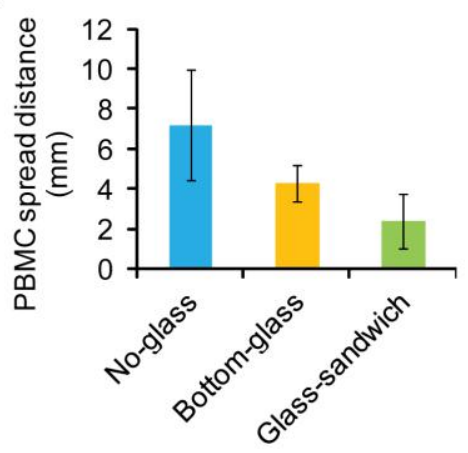

e

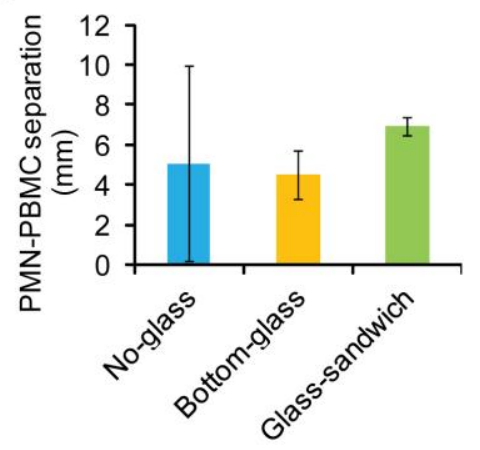

g

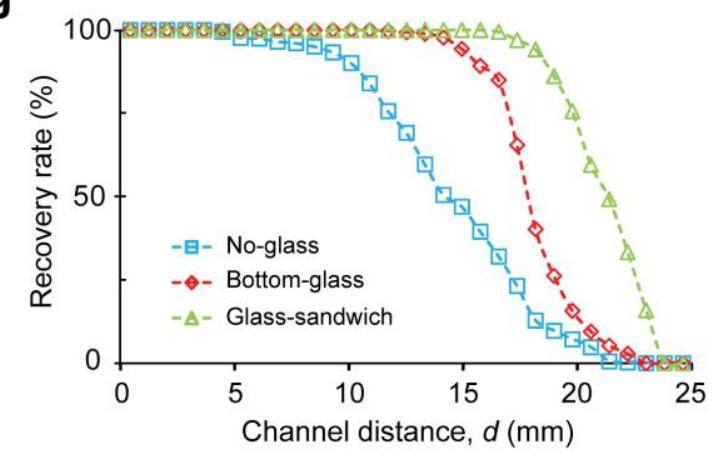

i

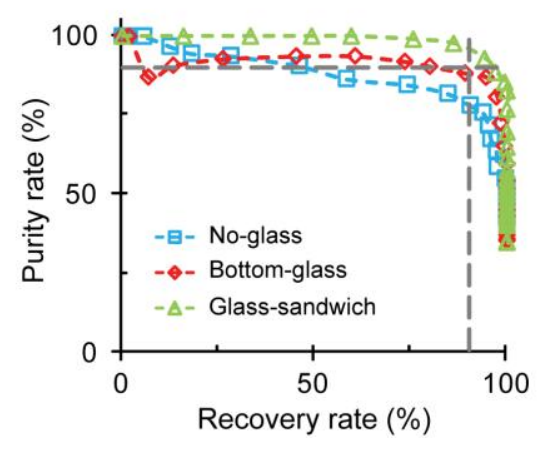



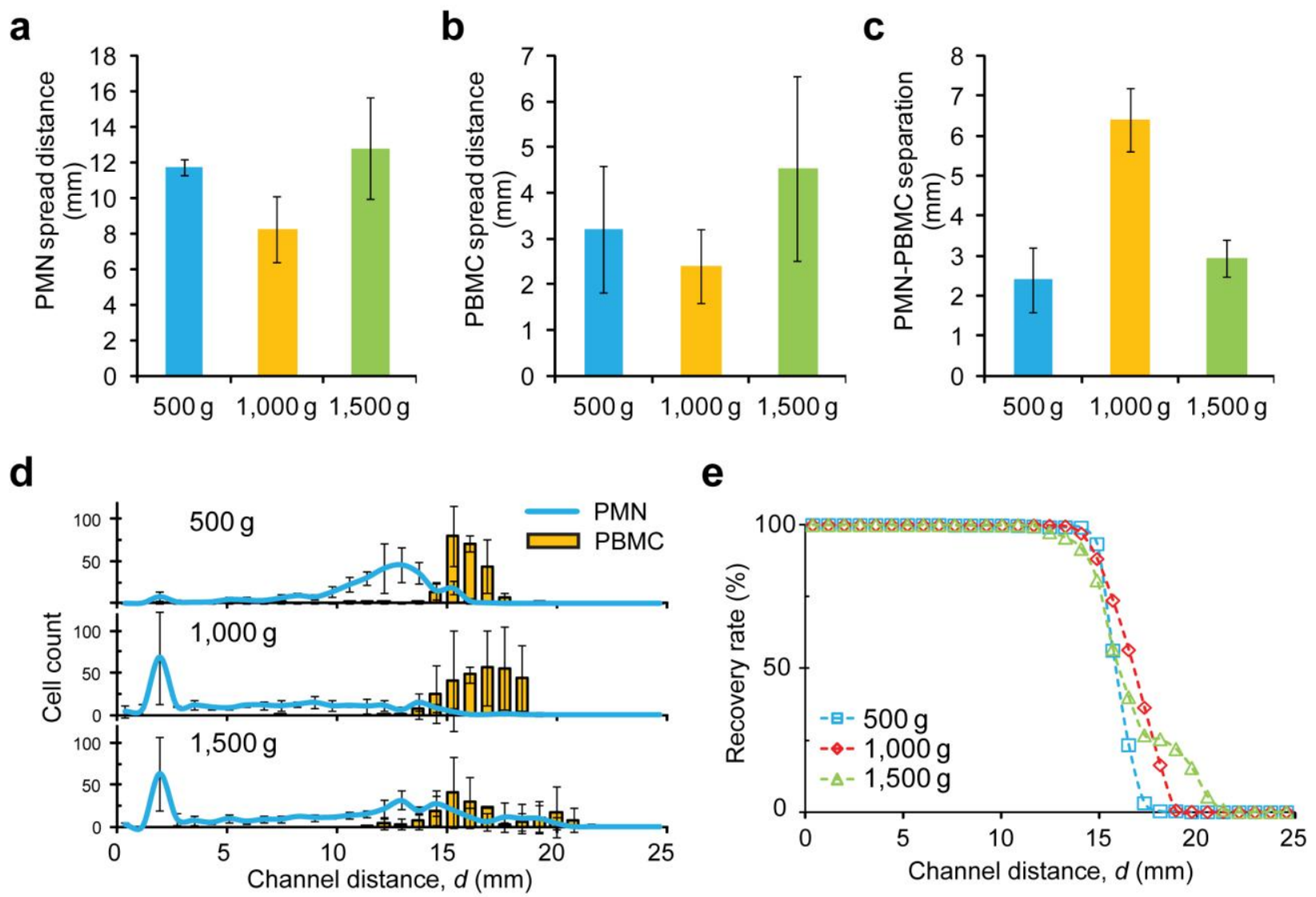

f

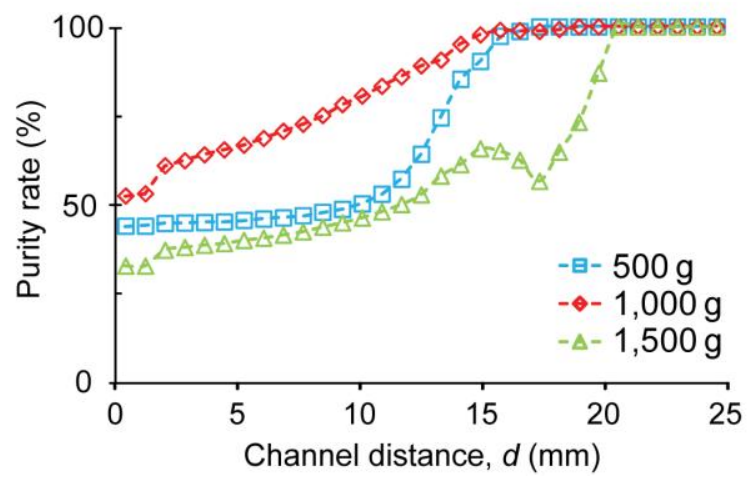

g

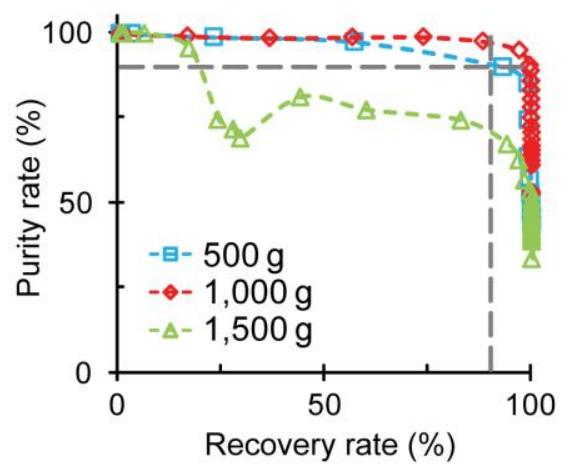


a

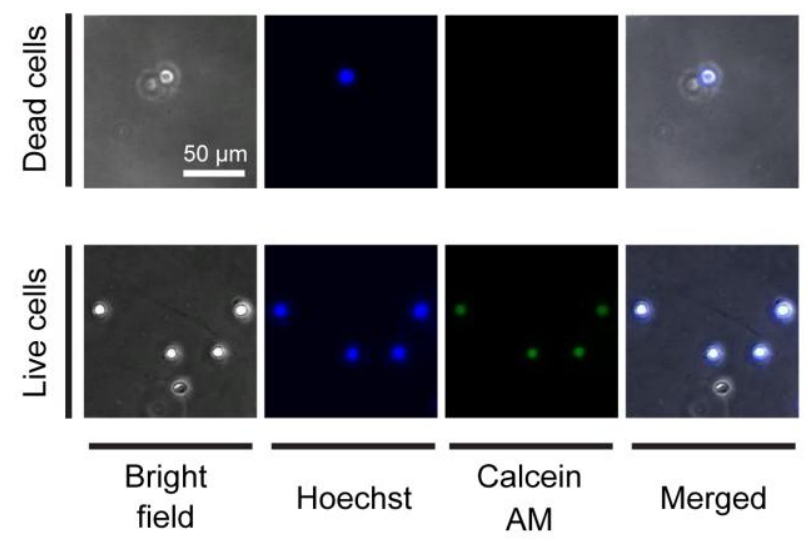

C

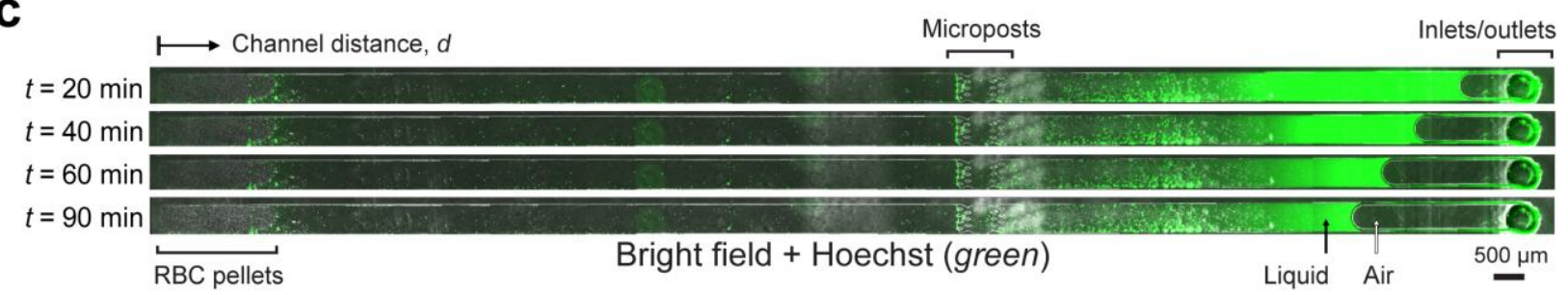

d

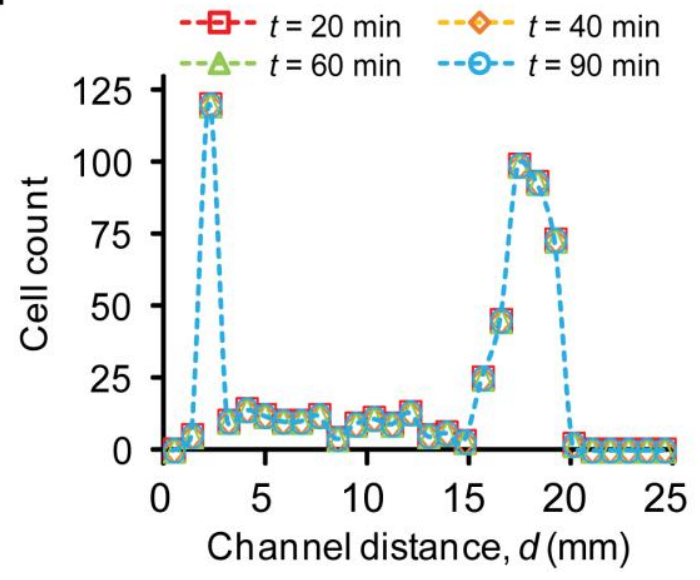

b

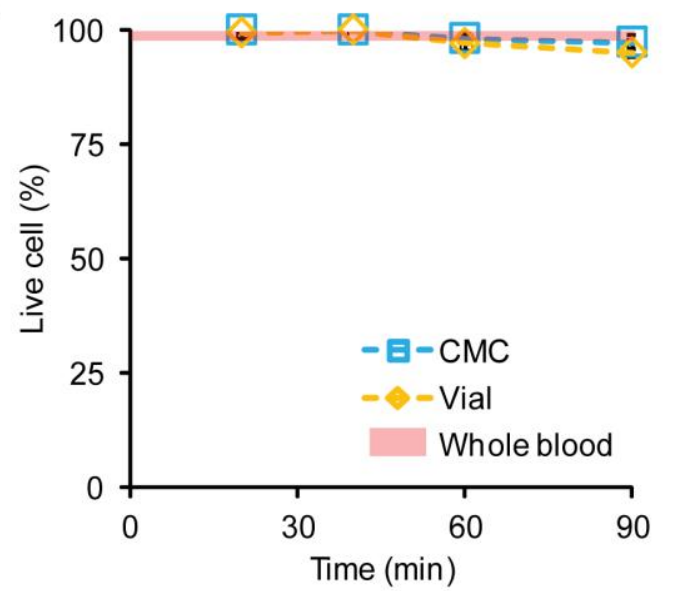

e
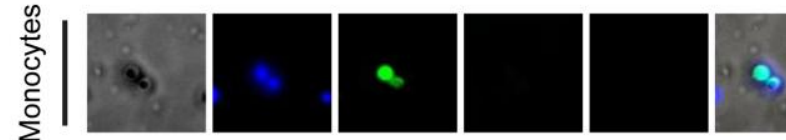

$\sum_{n}^{\infty}$
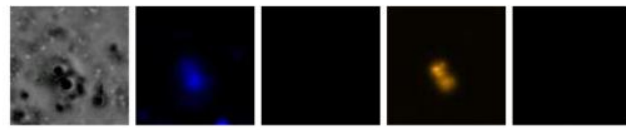

$\stackrel{\infty}{\overline{\mathcal{O}}}$
$\stackrel{\leftarrow}{\vdash}$
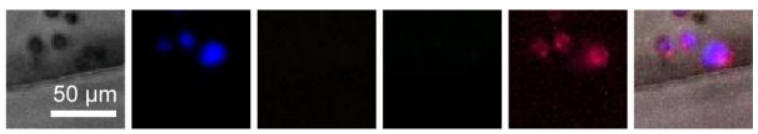

Bright field

Hoechst

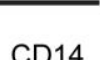

CD15

CD3 Merged 